\title{
Vladimir Vladimirovich Borodin (February 12, 1947, to March 23, 2010)
}

DOI: $10.1134 / \mathrm{S} 1063771010050258$

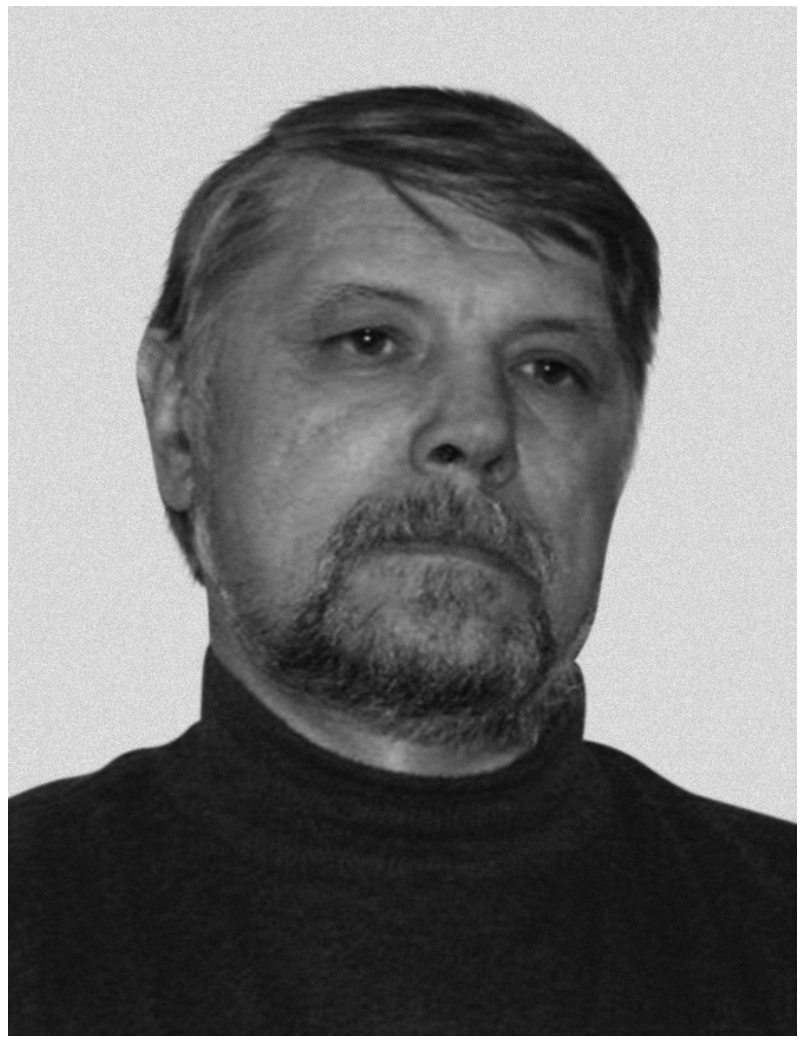

Domestic hydroacoustics has suffered a great loss. An outstanding scientist and specialist in the field of ocean acoustics and processing of hydroacoustic information, Dr. of Physicomathematical Sciences Vladimir Vladimirovich Borodin passed away on March 23, 2010.

V.V. Borodin was born on February 12, 1947. In 1971, after graduating from the Moscow Physical
Technical Institute, he began working at the N.N. Andreev Acoustics Institute. At the Acoustics Institute, he grew from a junior researcher to the head of a large scientific-research department. In 1979 he defended his Cand. Sci. Phys.-Math. Dissertation and in 1990 became a doctor of physicomathematical sciences.

In the period of work at the Acoustics Institute, Borodin carried out a whole series of first-class works on research into distribution and scattering of sound in the ocean. The original works of Borodin in the field of solving the direct and inverse problems of hydroacoustics have provided the basis for developing the compact computational procedures that have enabled the implementation of these methods in on-location hydroacoustic complexes to be started for the first time. The findings of Borodin's works in the field of the theory connected with the characteristics of the heterogeneous medium of processing hydroacoustic signals and noise have won renown and recognition in Russia and abroad.

In the recent years, Borodin successfully directed works on solving the problems of applied hydroacoustics at the A.M. Prokhorov Institute of General Physics, Russian Academy of Sciences. He headed the introduction of his theoretical and experimental developments into real complexes there. One of the complexes, the idea and operational principles of which personally belong to Borodin, has undergone state tests and is ready for serial production.

The significance of the results of Borodin's works for the country was marked by a Soviet State Award and multiple Russian Presidential Grants. The name of V.V. Borodin, who was a generously gifted, genuine, and principled man, will forever remain in domestic hydroacoustics and the memory of his friends and comrades-in-arms. 